\title{
Theory of staircase cyclic voltammetry of two electrode reactions coupled by a chemical reaction
}

\author{
Š. Komorsky-Lovrić, M. Lovrić \\ Divkovićeva 13, Zagreb 10090, Croatia
}

Received October 15, 2018, Accepted February 27, 2019

\begin{abstract}
Two reversible electrode reactions that are connected by either reversible or totally irreversible chemical reactions are theoretically analysed by staircase cyclic voltammetry. The dependence of peak potentials on the thermodynamic and kinetic parameters is calculated. If the mechanism is permanently in equilibrium, the stability constant of the reversible chemical reaction can be determined. Furthermore, the critical kinetic parameter is determined and its application to the measurement of the forward rate constant of the chemical reaction is demonstrated. Also, the influence of the kinetics of electrode reactions is discussed.
\end{abstract}

Keywords: ECE mechanism; Cyclic voltammetry; Theory

\section{INTRODUCTION}

The acronym ECE denotes an electrochemical mechanism in which two electrode reactions are coupled by a chemical reaction in the way that the product of the first electrode reaction is the reactant in a chemical reaction and the product of the latter is a reactant in a second electrode reaction [1-7]. This mechanism can be considered as a special case of the general nine-member square scheme [8-11]. It was observed in electroreduction of p-nitrosophenol [12, 13], carbonylmanganese compounds [14-16], benzenesulfonyl fluoride [17, 18], 1-butyl-3methylimidazolium bistriflimide [19], uranium complexes of acetylacetone [20], hexacyanochromate(III) [21], bimetallic organometallic complexes [22] and adriamycin [23], as well as in electrooxidation of tocopherols [24], copper(II) complex of thyrotropin-releasing hormone [25], methylcatechol [26, 27], dopamine [28] and aromatic hydrocarbons [29, 30]. The theory of the ECE mechanism is developed for chronoamperometry [31-33], d. c. polarography [34$36]$, linear scan [37, 38], cyclic [21,39] and square wave voltammetry [40-44], rotating disk [45] and ring-disk electrode measurements [46], the scanning electrochemical microscopy [47] and the surface reactions in protein film voltammetry [48, 49]. In this paper the main properties of responses of ECE mechanism in staircase cyclic voltammetry are recapitulated. The influences of both the thermodynamic constants under equilibrium conditions and the kinetic parameters of reversible and totally irreversible chemical reactions, as well as electrode reactions are considered.

\footnotetext{
* To whom all correspondence should be sent:

E-mail: mlovric@irb.hr
}

\section{MODELS}

The following reactions are investigated:

$$
\begin{aligned}
& \mathrm{A} \leftrightarrow \mathrm{B}^{+}+\mathrm{e}^{-} \\
& \mathrm{B}^{+}+\mathrm{X}^{-} \leftrightarrow \mathrm{E} \\
& \mathrm{E} \leftrightarrow \mathrm{F}^{+}+\mathrm{e}^{-}
\end{aligned}
$$

It is assumed that the mass transport can be described by the stationary, planar, semiinfinite diffusion model and that the reagent $\mathrm{X}^{-}$is present in great excess, so that the variation of its concentration can be neglected.

(1) The first model is based on the assumption that the chemical reaction is permanently in equilibrium. Its mathematical representation is given by the following differential equations and boundary conditions:

$$
\begin{aligned}
& \partial c_{A} / \partial t=D \partial^{2} c_{A} / \partial x^{2} \\
& \partial c_{\text {Int }} / \partial t=D \partial^{2} c_{\text {Int }} / \partial x^{2} \\
& \partial c_{F} / \partial t=D \partial^{2} c_{F} / \partial x^{2} \\
& c_{\text {Int }}=c_{B}+c_{E} \\
& \begin{array}{l}
t=0, \quad x \geq 0: \\
c_{X}=c_{X}^{*}
\end{array} \\
& \begin{array}{l}
t>0, x \rightarrow \infty: \\
0 c_{E} \rightarrow 0, c_{F} \rightarrow 0 \\
x=0:
\end{array} \quad c_{A} \rightarrow c_{A}^{*}, \quad c_{X} \rightarrow c_{X}^{*}, \quad c_{B} \rightarrow \\
& c_{B, x=0}=c_{A, x=0} \exp \left(F\left(E-E_{1}^{0}\right) / R T\right) \\
& K c_{X}^{*}=c_{E, x=0} / c_{B, x=0} \\
& c_{F, x=0}=c_{E, x=0} \exp \left(F\left(E-E_{2}^{0}\right) / R T\right)
\end{aligned}
$$


Š. Komorsky-Lovrić, M. Lovrić: Theory of staircase cyclic voltammetry of two electrode reactions ...

$$
\begin{aligned}
& D\left(\partial c_{A} / \partial x\right)_{x=0}=I_{1} / F S \\
& D\left(\partial c_{I n t} / \partial x\right)_{x=0}=\left(I_{2}-I_{1}\right) / F S \\
& D\left(\partial c_{F} / \partial x\right)_{x=0}=-I_{2} / F S \\
& x>0: K c_{X}^{*}=c_{E} / c_{B}
\end{aligned}
$$

\begin{tabular}{|c|c|}
\hline$\alpha_{1}, \alpha_{2}$ & $\begin{array}{l}\text { Transfer coefficients of the first and the } \\
\text { second electrode reactions }\end{array}$ \\
\hline$c_{A}, c_{B}, c_{E}, c_{F}$ & Concentrations of species A, $\mathrm{B}^{+}, \mathrm{E}$ and $\mathrm{F}^{+}$ \\
\hline$c_{A}^{*}, c_{X}^{*}$ & $\begin{array}{l}\text { Concentrations of species } \mathrm{A} \text { and } \mathrm{X}^{-} \text {in the } \\
\text { bulk of solution }\end{array}$ \\
\hline$D$ & Common diffusion coefficient \\
\hline E & Electrode potential \\
\hline$E_{1}^{0}, E_{2}^{0}$ & $\begin{array}{l}\text { Standard potentials of the first and the } \\
\text { second electrode reactions }\end{array}$ \\
\hline$\Delta E$ & $\begin{array}{l}\text { Potential step in staircase cyclic } \\
\text { voltammetry }\end{array}$ \\
\hline$E_{p, a}, E_{p, c}$ & Anodic and cathodic peak potentials \\
\hline$F$ & Faraday constant \\
\hline$I_{1}, I_{2}$ & $\begin{array}{l}\text { Currents of the first and the second electro } \\
\text { transfer }\end{array}$ \\
\hline K & $\begin{array}{l}\text { Equilibrium constant of the chemical } \\
\text { reactions }\end{array}$ \\
\hline$k$ & $\begin{array}{l}\text { Rate constant of irreversible chemical } \\
\text { reaction }\end{array}$ \\
\hline$k_{f}, k_{b}$ & $\begin{array}{l}\text { Rate constants of reversible chemical } \\
\text { reaction }\end{array}$ \\
\hline$k_{s 1}, k_{s 2}$ & $\begin{array}{l}\text { Rate constants of the first and the second } \\
\text { electrode reactions }\end{array}$ \\
\hline$R$ & Gas constant \\
\hline$S$ & Electrode surface area \\
\hline$T$ & Temperature \\
\hline$t$ & Time \\
\hline$v$ & Scan rate \\
\hline
\end{tabular}
1.

The meanings of symbols are reported in Table

Table 1. Meanings of symbols

Differential equations are solved by the numerical method [50, 51]. In staircase voltammetry the potential is changed in a stepwise manner and the scan rate is defined as the ratio of the height and the duration of the step: $v=\Delta E / \tau$. The current is measured at the end of each step. The dimensionless current is defined as follows: $\Phi_{k}=$ $I_{k}\left(F S c_{A}^{*}\right)^{-1}(D v F / R T)^{-1 / 2}$, where $k=1$ or 2 . In the simulation the time increment $d=\tau / 25$ and the fixed value $\Delta E=5 \mathrm{mV}$ were used. The numerical solution is the system of recursive formulae for the dimensionless current:

$\Phi_{2, m}=u_{1} u_{2}\left(1+u_{4}\right)^{-1}-\sum_{j=1}^{m-1} \Phi_{2, j} s_{m-j+1}$

$$
\begin{aligned}
& \Phi_{1, m}=\left(u_{1} u_{5}+\sum_{j=1}^{m} \Phi_{2, j} s_{m-j+1}\right)\left(1+u_{5}\right)^{-1}- \\
& \sum_{j=1}^{m-1} \Phi_{1, j} s_{m-j+1} \\
& u_{1}=5 \sqrt{\pi R T / F \Delta E} / 2 \\
& u_{2}= \\
& K c_{X}^{*} \exp \left(F\left(E-E_{1}^{0}\right) / R T\right) \exp \left(F\left(E-E_{2}^{0}\right) / R T\right)
\end{aligned}
$$

$$
\begin{aligned}
& u_{3}=K c_{X}^{*}\left[1+\exp \left(F\left(E-E_{2}^{0}\right) / R T\right)\right] \\
& u_{4}=\left(1+u_{3}\right) \exp \left(F\left(E-E_{1}^{0}\right) / R T\right) \\
& u_{5}=\left(1+K c_{X}^{*}\right) \exp \left(F\left(E-E_{1}^{0}\right) / R T\right) \\
& s_{p}=\sqrt{p}-\sqrt{p-1} \\
& m=1,2,3 \ldots
\end{aligned}
$$

The sum $\Phi=\Phi_{1}+\Phi_{2}$ is reported as a function of electrode potential.

(2) In the second model it is assumed that the chemical reaction is totally irreversible and of the first order:

$\mathrm{B}^{+} \rightarrow \mathrm{E}$

Consequently, equations (12) and (17) have to be replaced by the following equations:

$\partial c_{B} / \partial t=D \partial^{2} c_{B} / \partial x^{2}-k c_{B}$

$D\left(\partial c_{B} / \partial x\right)_{x=0}=-I_{1} / F S$

The following solution is obtained:

$\Phi_{1, m}=\left[u_{1} u_{6}-\kappa^{-1 / 2} \sum_{j=1}^{m-1} \Phi_{1, j} P_{m-j+1}-\right.$

$\left.u_{6} \sum_{j=1}^{m-1} \Phi_{1, j} s_{m-j+1}\right]\left(P_{1} \kappa^{-1 / 2}+u_{6}\right)^{-1}$

$\Phi_{2, m}=\left[\sum_{j=1}^{m} \Phi_{1, j} S_{m-j+1}-\right.$ $\left.u_{1} \kappa^{-1 / 2} \sum_{j=1}^{m} \Phi_{1, j} P_{m-j+1}\right] u_{7}-\sum_{j=1}^{m-1} \Phi_{2, j} s_{m-j+1}$

$u_{6}=\exp \left(F\left(E-E_{1}^{0}\right) / R T\right) / u_{1}$

$u_{7}=\exp \left(F\left(E-E_{2}^{0}\right) / R T\right) /[1+$ $\left.\exp \left(F\left(E-E_{2}^{0}\right) / R T\right)\right]$

$\kappa=k R T / F v$

$P_{i}=\operatorname{erf} \sqrt{\kappa q i}-\operatorname{erf} \sqrt{\kappa q(i-1)}$

$q=F \Delta E / 25 R T$

(3) The third model extends the second one by assuming that electrode reactions are not fast and reversible. Under this condition, equations (11) and (13) must be replaced by the following equations: 
Š. Komorsky-Lovrić, M. Lovrić: Theory of staircase cyclic voltammetry of two electrode reactions ...

$I_{1} / F S=-k_{S 1} \exp \left(-\alpha_{1} F\left(E-E_{1}^{0}\right) / R T\right)\left[c_{B, x=0}-\right.$

$\left.c_{A, x=0} \exp \left(F\left(E-E_{1}^{0}\right) / R T\right)\right]$

$I_{2} / F S=-k_{s 2} \exp \left(-\alpha_{2} F\left(E-E_{2}^{0}\right) / R T\right)\left[c_{F, x=0}-\right.$

$\left.c_{E, x=0} \exp \left(F\left(E-E_{2}^{0}\right) / R T\right)\right]$

The solution is as follows:

$\Phi_{1, m}=\left[u_{8}-u_{9} \sum_{j=1}^{m-1} \Phi_{1, j} P_{m-j+1}-\right.$

$\left.u_{8} u_{1}^{-1} \sum_{j=1}^{m-1} \Phi_{1, j} s_{m-j+1}\right] / u_{10}$

$\Phi_{2, m}=\left[u_{11} u_{1}^{-1} \sum_{j=1}^{m} \Phi_{1, j} s_{m-j+1}-\right.$

$u_{11} \kappa^{-1 / 2} \sum_{j=1}^{m} \Phi_{1, j} P_{m-j+1}-$

$\left.u_{12} u_{1}^{-1} \sum_{j=1}^{m-1} \Phi_{2, j} s_{m-j+1}\right] /\left(1+u_{12} u_{1}^{-1}\right)$

$u_{8}=\lambda_{1} \exp \left(\left(1-\alpha_{1}\right) F\left(E-E_{1}^{0}\right) / R T\right)$

$u_{9}=\lambda_{1} \exp \left(-\alpha_{1} F\left(E-E_{1}^{0}\right) / R T\right) \kappa^{-1 / 2}$

$u_{10}=1+u_{9} P_{1}+u_{8} / u_{1}$

$u_{11}=\lambda_{2} \exp \left(\left(1-\alpha_{2}\right) F\left(E-E_{2}^{0}\right) / R T\right)$

$u_{12}=\lambda_{2} \exp \left(-\alpha_{2} F\left(E-E_{2}^{0}\right) / R T\right)[1+$ $\left.\exp \left(F\left(E-E_{2}^{0}\right) / R T\right)\right]$

$\lambda_{1}=k_{s 1}(D F v / R T)^{-1 / 2}$

$\lambda_{2}=k_{s 2}(D F v / R T)^{-1 / 2}$

(4) The last model is a general solution for two reversible electrode reactions coupled by a kinetically controlled reversible chemical reaction. In this case equations (12) and (17) must be replaced by the following equations:

$$
\begin{gathered}
c_{H}=K c_{X}^{*} c_{B}-c_{E} \\
\partial c_{H} / \partial t=D \partial^{2} c_{H} / \partial x^{2}-\varepsilon c_{H} \\
D\left(\partial c_{H} / \partial x\right)_{x=0}=-K c_{X}^{*} I_{1} / F S-I_{2} / F S \\
\varepsilon=k_{b}\left(1+K c_{X}^{*}\right) \\
K=k_{f} / k_{b}
\end{gathered}
$$

The solution is given by the following recursive formulae:

$$
\begin{aligned}
& \Phi_{2, m}=-z_{8} z_{7}^{-1}+z_{9} z_{7}^{-1} \sum_{j=1}^{m-1} \Phi_{2, j} S_{m-j+1}+ \\
& z_{10} z_{7}^{-1} \sum_{j=1}^{m-1} \Phi_{2, j} P_{m-j+1}+ \\
& z_{11} z_{7}^{-1} \sum_{j=1}^{m-1} \Phi_{1, j} S_{m-j+1}+ \\
& z_{12} z_{7}^{-1} \sum_{j=1}^{m-1} \Phi_{1, j} P_{m-j+1} \\
& \Phi_{1, m}=z_{8}+z_{1} z_{5}^{-1} \sum_{j=1}^{m} \Phi_{2, j} S_{m-j+1}- \\
& z_{2} z_{5}^{-1} \sum_{j=1}^{m} \Phi_{2, j} P_{m-j+1}-z_{13} \sum_{j=1}^{m-1} \Phi_{1, j} S_{m-j+1}- \\
& z_{3} z_{5}^{-1} \sum_{j=1}^{m-1} \Phi_{1, j} P_{m-j+1}
\end{aligned}
$$

$$
\begin{aligned}
& z_{1}=u_{1}^{-1}\left(1+K c_{X}^{*}\right)^{-1} \\
& z_{2}=\left(1+K c_{X}^{*}\right)^{-3 / 2} \kappa_{b}^{-1 / 2} \\
& z_{3}=K c_{X}^{*} z_{2} \\
& z_{4}=K c_{X}^{*} z_{1} \\
& z_{5}=z_{1}+u_{1}^{-1} \exp \left(F\left(E-E_{1}^{0}\right) / R T\right)+z_{3} P_{1} \\
& z_{6}=\left[z_{4}+z_{3} P_{1}\right] \exp \left(F\left(E-E_{2}^{0}\right) / R T\right) \\
& z_{7}=z_{5}^{-1}\left(z_{1}-z_{2}\right)-z_{6}^{-1}\left[u_{1}^{-1}+\left(z_{4}+\right.\right. \\
& \left.\left.\left.z_{2} P_{1}\right) \exp ^{-1}\left(E-E_{2}^{0}\right) / R T\right)\right] \\
& z_{8}=z_{5}^{-1} \exp \left(F\left(E-E_{1}^{0}\right) / R T\right) \\
& z_{9}=u_{1}^{-1} z_{6}^{-1}+z_{4} z_{6}^{-1} \exp \left(F\left(E-E_{2}^{0}\right) / R T\right)- \\
& z_{1} z_{5}^{-1} \\
& z_{10}=z_{2} z_{6}^{-1} \exp \left(F\left(E-E_{2}^{0}\right) / R T\right)+z_{2} z_{5}^{-1} \\
& z_{11}=z_{13}-z_{4} z_{6}^{-1} \exp \left(F\left(E-E_{2}^{0}\right) / R T\right) \\
& z_{12}=z_{3} z_{6}^{-1} \exp \left(F\left(E-E_{2}^{0}\right) / R T\right)+z_{3} z_{5}^{-1}
\end{aligned}
$$$$
z_{13}=\left[z_{1}+u_{1}^{-1} \exp \left(F\left(E-E_{1}^{0}\right) / R T\right)\right] / z_{5}
$$$$
\kappa_{b}=k_{b} R T / F v
$$$$
P_{i}=\operatorname{erf} \sqrt{\kappa_{b}\left(1+K c_{X}^{*}\right) q i}-
$$$$
\operatorname{erf} \sqrt{\kappa_{b}\left(1+K c_{X}^{*}\right) q(i-1)}
$$

\section{RESULTS AND DISCUSSION}

Staircase cyclic voltammograms of the mechanisms (1) - (3) under equilibrium conditions depend on the standard potentials of electron transfers and the dimensionless constant $K c_{X}^{*}$ of the chemical reaction. We shall investigate an ideal case in which the concentration of reagent $\mathrm{X}^{-}$can be changed experimentally from zero to the limit of solubility. If $E_{1}^{0}=E_{2}^{0}$ and $K c_{X}^{*}=1$, the response exhibits a single pair of peaks with the dimensionless peak currents $\Phi_{p, a}=0.773$ and $\Phi_{p, c}$ $=-0.574$ that appear at $E_{p, a}=0.035 \mathrm{~V} v s . E_{1}^{0}$ and $E_{p, c}-E_{1}^{0}=-0.040 \mathrm{~V}$. This is shown in Fig. 1. The real peak currents depend linearly on the square root of scan rate, but the peak potentials are independent of the scan rate. If the concentration of $\mathrm{X}^{-}$is reduced to zero, only the first electrode reaction occurs and the peak currents are diminished to 0.3865 and 0.287 , but the peak potentials do not change. The peak separation is $75 \mathrm{mV}$ and the mid-potential is 
Š. Komorsky-Lovrić, M. Lovrić: Theory of staircase cyclic voltammetry of two electrode reactions ...

close to the first standard potential. The difference of $-2.5 \mathrm{mV}$ is the consequence of changing the potential in discrete quantity $\Delta E$.

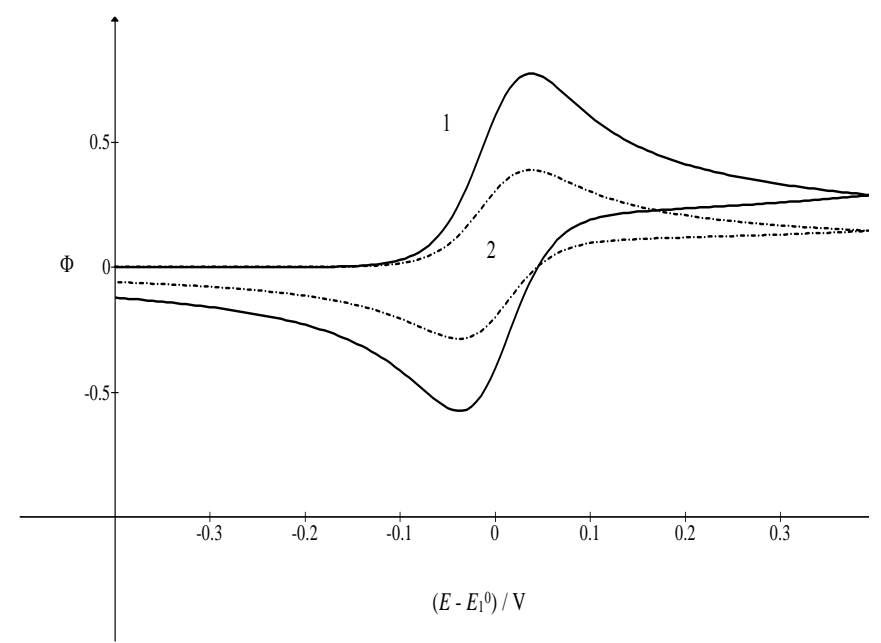

Fig. 1. Dimensionless staircase cyclic voltammograms of ECE mechanism under equilibrium conditions (eqs. 18 and 19). $\Delta E=5 \mathrm{mV}, E_{2}^{0}=E_{1}^{0}$ and $K c_{X}^{*}=1$ (1) and 0 (2).

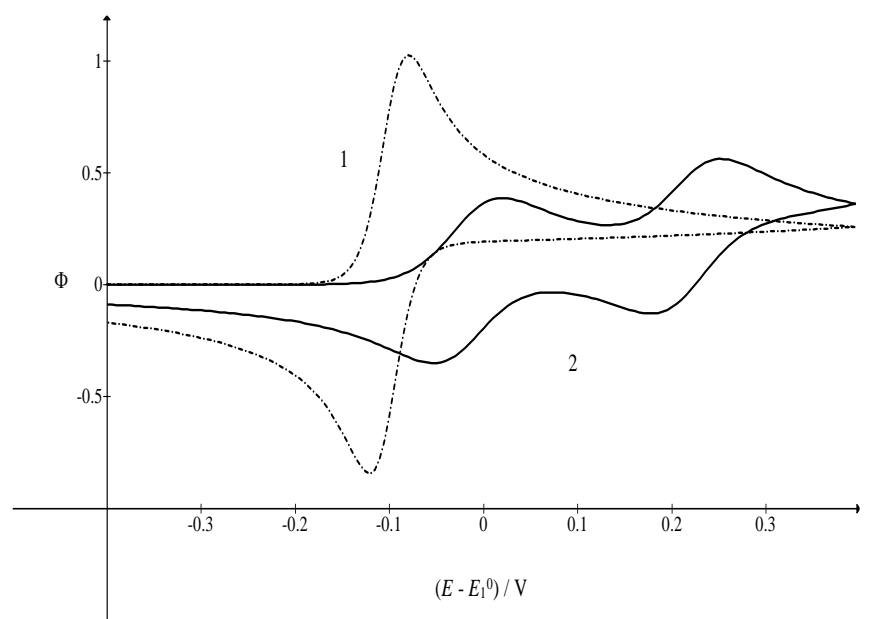

Fig. 2. Dimensionless staircase cyclic voltammograms of ECE mechanism under equilibrium conditions. $K c_{X}^{*}=$ 1 and $\left(E_{2}^{0}-E_{1}^{0}\right) / \mathrm{V}=-0.2(1)$ and $0.2(2)$.

Fig. 2 shows the influence of the difference in standard potentials on cyclic voltammograms. If $E_{2}^{0}-E_{1}^{0}=-0.2 \mathrm{~V}$, the intermediate is unstable and only two peaks appear: $\Phi_{p, a}=1.023$ and $\Phi_{p, c}=$ 0.844 . The peak potentials are $E_{p, a}-E_{1}^{0}=-0.080 \mathrm{~V}$ and $E_{p, c}-E_{1}^{0}=-0.120 \mathrm{~V}$. In the case of stable intermediate, the voltammogram consists of two pairs of peaks: $\Phi_{p, 1, a}=0.387$ and $\Phi_{p, 1, c}=-0.350$, appearing at $E_{p, 1, a}-E_{1}^{0}=0.020 \mathrm{~V}$ and $E_{p, 1, c}-E_{1}^{0}$ $=-0.055 \mathrm{~V}$, and $\Phi_{p, 2, a}=0.562$ and $\Phi_{p, 2, c}=-0.128$ that appear at $E_{p, 2, a}-E_{1}^{0}=0.250 \mathrm{~V}$ and $E_{p, 2, c}-E_{1}^{0}$ $=0.180 \mathrm{~V}$. Table 2 reports the peak potentials as a function of the difference between standard potentials.
Table 2. Dependence of anodic and cathodic peak potentials on the difference between standard potentials for $K c_{X}^{*}=1$.

\begin{tabular}{|c|c|c|c|c|}
\hline$\left(\begin{array}{c}E_{2}^{0}- \\
\left.E_{1}^{0}\right) / \mathrm{V}\end{array}\right.$ & $\begin{array}{c}E_{p, 1, a} / \\
\mathrm{V}\end{array}$ & $\begin{array}{c}E_{p, 1, c} / \\
\mathrm{V}\end{array}$ & $\begin{array}{c}E_{p, 2, a} / \\
\mathrm{V}\end{array}$ & $\begin{array}{c}E_{p, 2, c} / \\
\mathrm{V}\end{array}$ \\
\hline-0.300 & -0.130 & -0.170 & & \\
\hline-0.250 & -0.105 & -0.145 & & \\
\hline-0.200 & -0.080 & -0.120 & & \\
\hline-0.150 & -0.055 & -0.095 & & \\
\hline-0.100 & -0.030 & -0.075 & & \\
\hline-0.050 & 0.000 & -0.050 & & \\
\hline 0.000 & 0.035 & -0.040 & & \\
\hline 0.050 & 0.090 & -0.045 & & \\
\hline 0.060 & 0.105 & -0.045 & & \\
\hline 0.070 & 0.115 & -0.045 & & \\
\hline 0.080 & 0.125 & -0.045 & & \\
\hline 0.090 & 0.135 & -0.050 & & \\
\hline 0.100 & 0.030 & -0.050 & 0.150 & - \\
\hline 0.150 & 0.020 & -0.050 & 0.200 & 0.125 \\
\hline 0.200 & 0.020 & -0.055 & 0.250 & 0.180 \\
\hline 0.250 & 0.020 & -0.055 & 0.305 & 0.230 \\
\hline 0.300 & 0.020 & -0.055 & 0.355 & 0.280 \\
\hline
\end{tabular}

If $E_{2}^{0}-E_{1}^{0}<-0.1 \mathrm{~V}$, the peak separation is 40 $\mathrm{mV}$ and the mid-potential is equal to the average of standard potentials. The latter is in agreement with the properties of the simple EE mechanism [52 54]. The peak separation increases to $75 \mathrm{mV}$ for $E_{2}^{0}=E_{1}^{0}$ and to $185 \mathrm{mV}$ for $E_{2}^{0}-E_{1}^{0}=0.090 \mathrm{~V}$. This is the consequence of the development of the second pair of peaks, as can be seen in Fig. 3. The response is split it two pairs of peaks if $E_{2}^{0}-E_{1}^{0}>$ $0.1 \mathrm{~V}$. The peak separations are $75 \mathrm{mV}$ and the midpotentials are equal to $E_{1}^{0}-17.5 \mathrm{mV}$ and $E_{2}^{0}+17.5$ $\mathrm{mV}$. In the further analysis the mid-potential will be neglected.

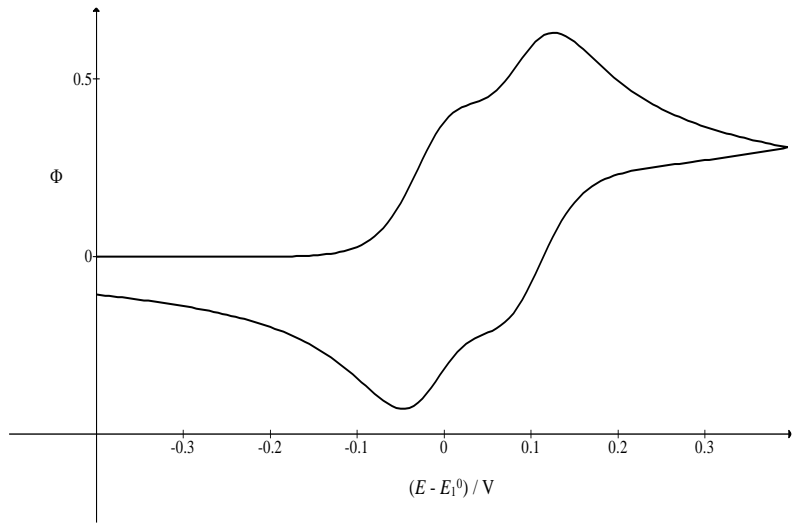

Fig. 3. $\mathrm{CV}$ of ECE in the equilibrium. $K c_{X}^{*}=1$ and $E_{2}^{0}-E_{1}^{0}=0.080 \mathrm{~V}$.

The second variable that governs the response is the dimensionless constant $K c_{X}^{*}$. An example is shown in Fig. 4. 
Š. Komorsky-Lovrić, M. Lovrić: Theory of staircase cyclic voltammetry of two electrode reactions ...

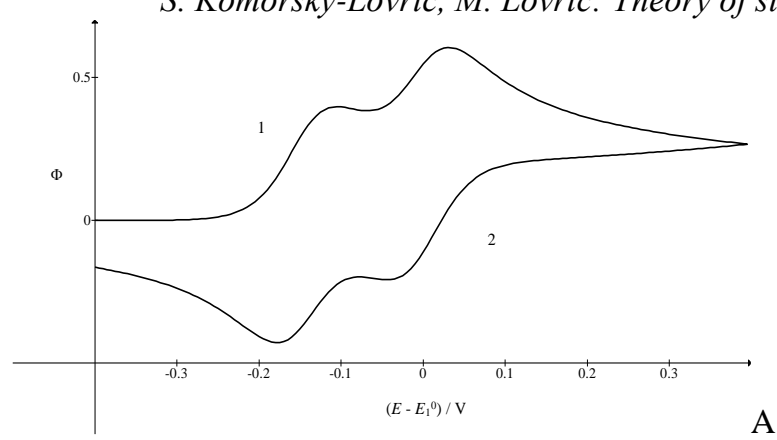

A

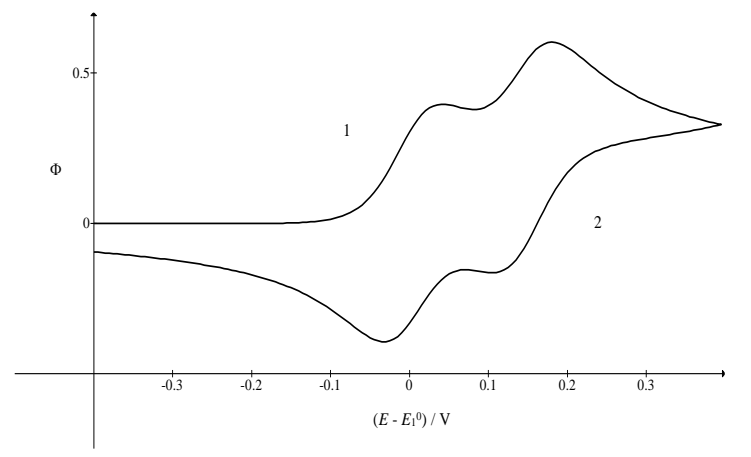

B

Fig. 4. $\mathrm{CV}$ of $\mathrm{ECE}$ in the equilibrium. $E_{2}^{0}=E_{1}^{0}$ and $K c_{X}^{*}=300(\mathrm{~A})$ and $0.003(\mathrm{~B})$. Two pairs of peaks are marked.

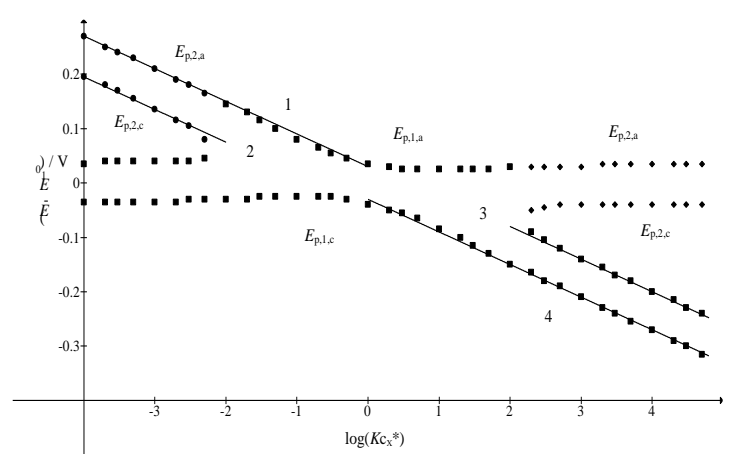

Fig. 5. Relationship between peak potentials in CV and the logarithm of dimensionless equilibrium constant of chemical reaction. ECE in the permanent equilibrium. $E_{2}^{0}=E_{1}^{0}$. The straight lines are linear approximations.

If $E_{2}^{0}=E_{1}^{0}$ and $K c_{X}^{0}=300$, the first electron transfer occurs at the potential that is lower than the standard potential $\left(E_{p, 1, a}-E_{1}^{0}=-0.105 \mathrm{~V}\right.$ and $\left.E_{p, 1, c}-E_{1}^{0}=-0.180 \mathrm{~V}\right)$ while the second charge transfer remains unchanged $\left(E_{p, 2, a}-E_{1}^{0}=0.030 \mathrm{~V}\right.$ and $\left.E_{p, 2, c}-E_{1}^{0}=-0.045 \mathrm{~V}\right)$. This is because the chemical reaction consumes the product of the first electrode reaction and decreases the formal potential of this reaction.

The situation is opposite if $K c_{X}^{*}=0.003$ and the chemical equilibrium is shifted towards $\mathrm{B}^{+}$species. In this case additional energy is required to surmount the chemical reaction preceding the second charge transfer [55]. For this reason the peak potentials corresponding to the second electrode reaction are higher than the second standard potential: $E_{p, 2, a}-E_{1}^{0}=0.180 \mathrm{~V}$ and $E_{p, 2, c}-E_{1}^{0}=$ $0.105 \mathrm{~V}$. These explanations are confirmed by the dependence of anodic and cathodic peak potentials on the logarithm of the product $K c_{X}^{*}$ that is shown in Fig. 5 for $E_{2}^{0}=E_{1}^{0}$. Under this condition the cyclic voltammogram splits in two pairs of peaks if either $K c_{X}^{*}>100$ or $K c_{X}^{*}<0.01$. Within these boundaries only two peaks appear, but their separation increases from $75 \mathrm{mV}$ for $K c_{X}^{*}=1$ to $110 \mathrm{mV}$ for $K c_{X}^{*}=10$, or $K c_{X}^{*}=0.1$, and to $180 \mathrm{mV}$ for $K c_{X}^{*}=100$ or $K c_{X}^{*}=$ 0.01 . In the split responses one pair of peak potentials depends linearly on the logarithm of $K c_{X}^{*}$ while the other pair is independent of this variable. The straight lines in Fig. 5 are defined by the following equations: $E_{p, 2, a}-E_{1}^{0}=-2.3(R T / F)$ $\log \left(K c_{X}^{*}\right)+0.030 \mathrm{~V}(1), E_{p, 2, c}-E_{1}^{0}=-2.3(R T / F)$ $\log \left(K c_{X}^{*}\right)-0.045 \mathrm{~V}(2), E_{p, 1, a}-E_{1}^{0}=-2.3(R T / F)$ $\log \left(K c_{X}^{*}\right)+0.040 \mathrm{~V}$ (3) and $E_{p, 1, c}-E_{1}^{0}=-2.3$ $(R T / F) \log \left(K c_{X}^{*}\right)-0.030 \mathrm{~V}(4)$. One can see that the equilibrium constant $K$ can be estimated if the product $K c_{X}^{*}$ can be changed either from 0.1 to 100 , or from 0.01 to 10 . In the first case the cathodic peak potential changes from $E_{p, 1, c}-E_{1}^{0}=-0.030 \mathrm{~V}$ to the straight line 4 . The intersection of these two lines reveals the equilibrium constant: $\log \left(K c_{X}^{*}\right)_{\text {cross }}=0$ and $K=\left(c_{X}^{*}\right)_{\text {cross. }}^{-1}$ The same applies to the intersection of the straight line $E_{p, 2, a}-E_{1}^{0}=0.030$ $\mathrm{V}$ and the straight line 1 . In experiments the condition $c_{X}^{*} \gg c_{A}^{*}$ must be satisfied.

The relationship between peak potentials and the equilibrium constant depends on the difference in standard potentials. This is shown in Figs. 6 and 7.

If two pairs of peaks appear at all values of $\log \left(K c_{X}^{*}\right)$, the first electrode reaction is influenced by the chemical reaction if $K c_{X}^{*}>0.1$, while the peak potentials of the second electrode reaction depend on $\log \left(K c_{X}^{*}\right)$ if it is smaller than 1 . The straight lines 1 and 2 in Fig. 6 satisfy the equations: $E_{p, 2, a}-E_{1}^{0}=-2.3(R T / F) \log \left(K c_{X}^{*}\right)+E_{2}^{0}-E_{1}^{0}+$ $0.035 \mathrm{~V}$ and $E_{p, 2, c}-E_{1}^{0}=-2.3(R T / F) \log \left(K c_{X}^{*}\right)+$ $E_{2}^{0}-E_{1}^{0}-0.040 \mathrm{~V}$. If $K c_{X}^{*}>10$ the peak potentials acquire constant values $E_{p, 2, a}-E_{1}^{0}=E_{2}^{0}-E_{1}^{0}+$ $0.035 \mathrm{~V}$ and $E_{p, 2, c}-E_{1}^{0}=E_{2}^{0}-E_{1}^{0}-0.040 \mathrm{~V}$. These relationships can be used for the calculation of equilibrium constant. The lines 3 and 4 in Figs. 5 and 6 are identical. 
Š. Komorsky-Lovrić, M. Lovrić: Theory of staircase cyclic voltammetry of two electrode reactions ...

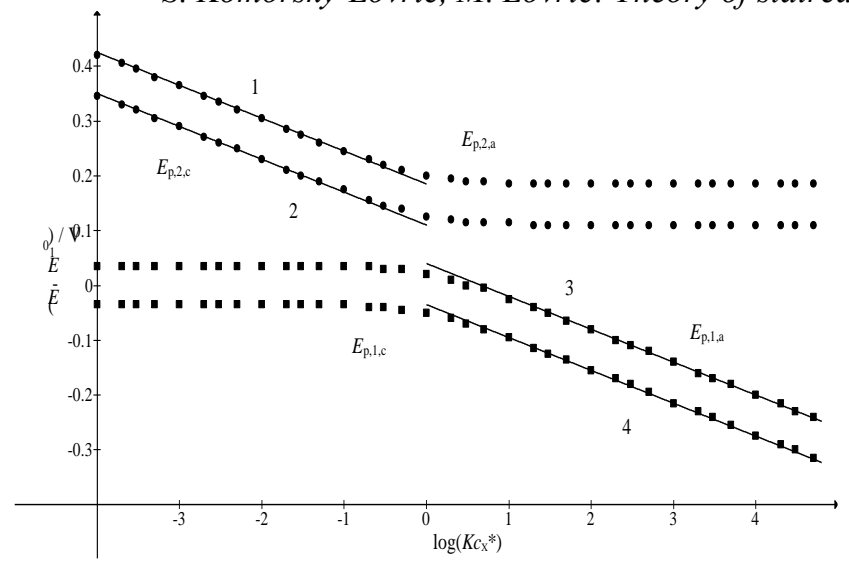

Fig. 6. Dependence of peak potentials on the logarithm of the product $K c_{X}^{*} . E_{2}^{0}-E_{1}^{0}=0.150 \mathrm{~V}$.

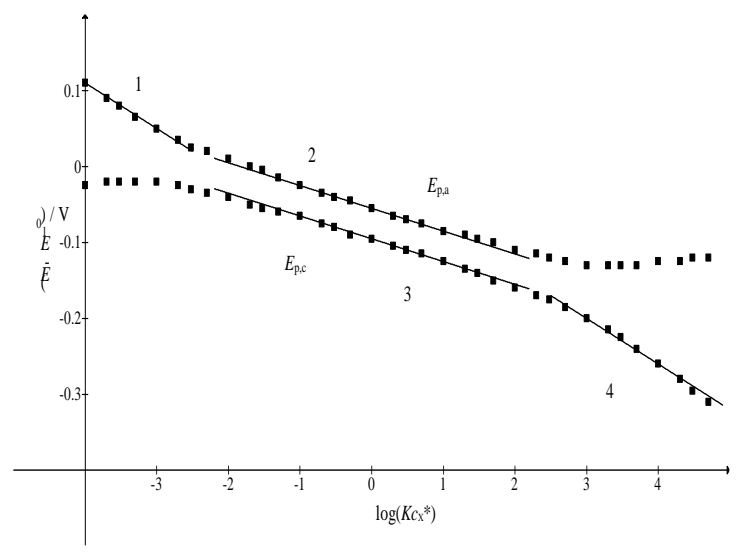

Fig. 7. Dependence of peak potentials on the logarithm of the product $K c_{X}^{*} . E_{2}^{0}-E_{1}^{0}=-0.150 \mathrm{~V}$.

Fig. 7 shows that for $E_{2}^{0}-E_{1}^{0}=-0.150 \mathrm{~V}$ a single pair of peaks appears within a wide range $-4<$ $\log \left(K c_{X}^{*}\right)<5$. The peak separation is constant if $-1<$ $\log \left(K c_{X}^{*}\right)<1$ and within these boundaries the slopes of linear dependence of peak potentials on the logarithm of $K c_{X}^{*}$ are equal to $-0.030 \mathrm{~V}$. This slope corresponds to the average of the formal potentials of the first and the second electrode reaction:

$\bar{E}=\left[E_{1}^{0}-(R T / F) \ln \left(K c_{X}^{*}\right)+E_{2}^{0}\right] / 2$

So, the straight lines 2 and 3 are defined by the equations:

$E_{p, a}-E_{1}^{0}=-2.3(R T / 2 F) \log \left(K c_{X}^{*}\right)+\left(E_{2}^{0}-E_{1}^{0}\right) / 2$ $+0.020 \mathrm{~V}$

$E_{p, c}-E_{1}^{0}=-2.3(R T / 2 F) \log \left(K c_{X}^{*}\right)+\left(E_{2}^{0}-E_{1}^{0}\right) / 2-$ $0.020 \mathrm{~V}$

For the biggest and the smallest values of $K c_{X}^{*}$ the shoulders develop, as in Fig. 3, and the responses start to split. The slopes of straight lines 1 and 4 are $-2.3(R T / F)$, as in CE and EC mechanisms.
The figures 5, 6 and 7 show that the form of response depends on the product $K c_{X}^{*}$ and the difference in standard potentials. One may define a critical value of $K c_{X}^{*}$ above which the voltammogram consists of two pairs of peaks. Our calculation shows that the logarithm of this critical value is a linear function of the difference between standard potentials expressed in volts: $\log \left(K c_{X}^{*}\right)_{\text {crit. }}=$ $-17.16\left(E_{2}^{0}-E_{1}^{0}\right)+2.255$.

Fig. 8 shows the influence of the kinetics of irreversible chemical reaction on the response of ECE mechanism. Dimensionless voltammograms depend on the dimensionless rate constant $\kappa=$ $k R T / F v$. If the intermediate is stable, the second pair of peaks develops with the increasing rate constant, but the first cathodic peak is diminished. If $\kappa=0.01$ there is no second pair of peaks and the response is characterised by $\Phi_{p, a}=0.387, E_{p, a}-E_{1}^{0}$ $=0.035 \mathrm{~V}, \Phi_{p, c}=-0.242$ and $E_{p, c}-E_{1}^{0}=-0.035 \mathrm{~V}$. For $\kappa=0.001$ the anodic branch does not change, but the cathodic peak current decreases to -0.287 and appears at $-0.040 \mathrm{~V} v s$. $E_{1}^{0}$. This is identical to the curve 2 in Fig. 1. If the rate constant is increased to 1 , the following characteristic currents and potentials are obtained: $\Phi_{p, 1, a}=0.421, E_{p, 1, a}-E_{1}^{0}$ $=0.020 \mathrm{~V}, \Phi_{p, 1, c}=-0.060, E_{p, 1, c}-E_{1}^{0}=-0.075 \mathrm{~V}$, $\Phi_{p, 2, a}=0.504, E_{p, 2, a}-E_{1}^{0}=0.235 \mathrm{~V}, \Phi_{p, 2, c}=$ 0.142 and $E_{p, 2, c}-E_{1}^{0}=0.160 \mathrm{~V}$. The response of the second electrode reaction is similar to the curve 2 in Fig. 2, but the first electrode reaction appears totally irreversible. This is because the product $\mathrm{B}^{+}$is irreversibly transformed into the species $\mathrm{E}$ and cannot be reduced back to the reactant $\mathrm{A}$.

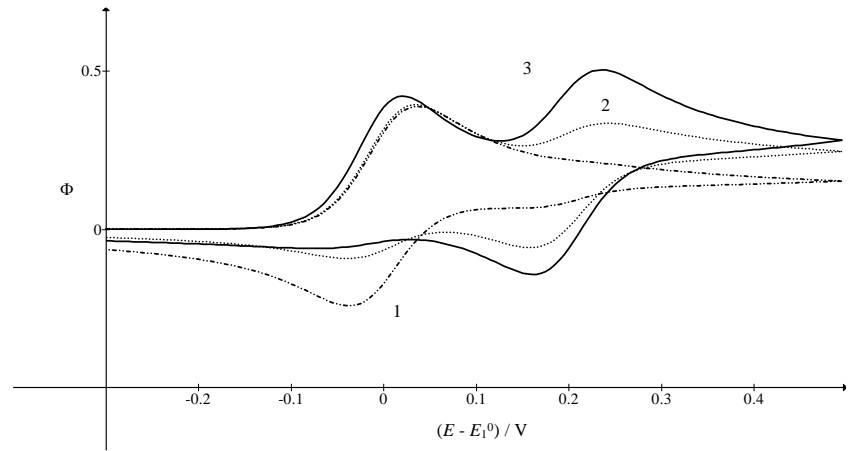

Fig. 8. Dimensionless staircase cyclic voltammograms of ECE mechanism influenced by the kinetics of totally irreversible chemical reaction (eqs. 30 and 31). $\Delta E=5$ $\mathrm{mV}, E_{2}^{0}-E_{1}^{0}=0.2 \mathrm{~V}$ and $\kappa=0.01$ (1), 0.1 (2) and 1 (3).

Fig. 9 shows the relationship between dimensionless peak currents and peak potentials and the logarithm of dimensionless rate constant. One can notice that the second anodic peak appears for $\kappa_{\text {crit }}=0.03$. If the parameter $\kappa$ is changed by the variation of scan rate, the real rate constant is related to the critical scan rate, at which the second peak 


\section{$\check{S}$. Komorsky-Lovrić, M. Lovrić: Theory of staircase cyclic voltammetry of two electrode reactions ...} appears, by the following equations: $k=$ $\kappa_{\text {crit }} v_{\text {crit }} /(R T / F)$ and $k=1.17 v_{\text {crit }}$.

Fig. 10 shows a simulation of a real experiment in which the critical scan rate is determined. The second anodic peak can be observed below $v=0.1$ $\mathrm{V} / \mathrm{s}$ and disappears for higher scan rates. This suggests that $k=0.117 \mathrm{~s}^{-1}$. The rate constant influences mainly the peak potentials of the first electrode reaction, as can be seen in Fig. 9B.

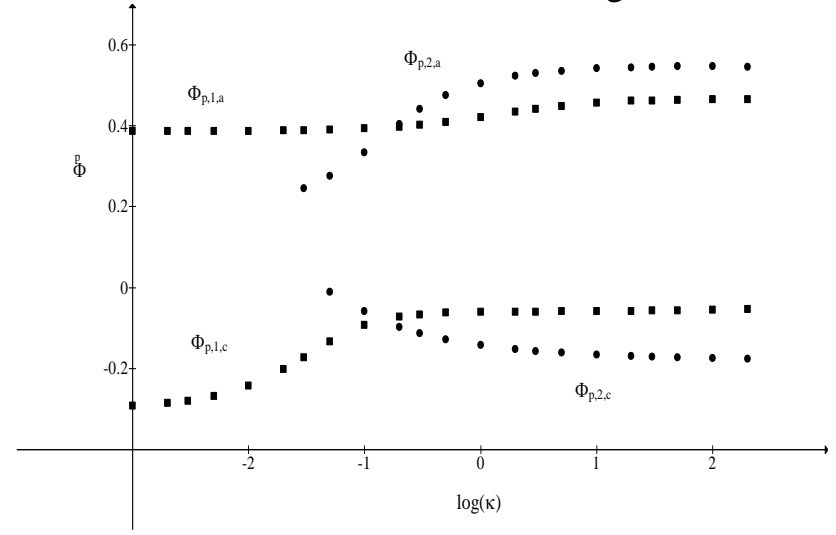

A

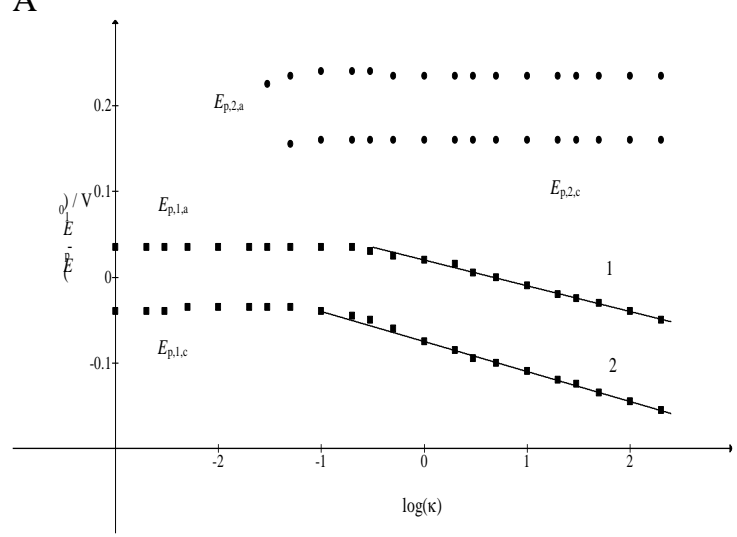

B

Fig. 9. Dependence of dimensionless peak currents (A) and peak potentials (B) on the logarithm of dimensionless rate constant of irreversible chemical reaction. $E_{2}^{0}-E_{1}^{0}=0.2 \mathrm{~V}$.

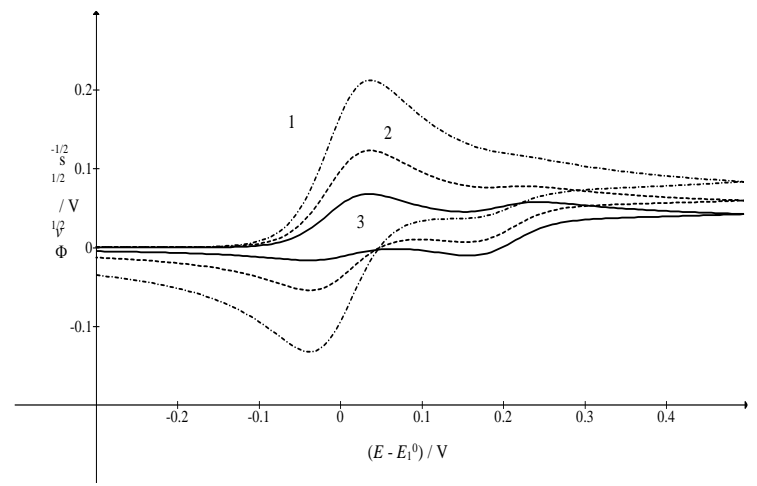

Fig. 10. Normalized cyclic voltammograms of ECE mechanism controlled by the chemical kinetics. $E_{2}^{0}-E_{1}^{0}$ $=0.2 \mathrm{~V}, k=0.117 \mathrm{~s}^{-1}$ and $v /(\mathrm{V} / \mathrm{s})=0.3(1), 0.1$ (2) and $0.03(3)$.
The straight lines 1 and 2 satisfy the following equations: $E_{p, 1, a}-E_{1}^{0}=-0.030 \log (\kappa)+0.020 \mathrm{~V}$ and $E_{p, 1, c}-E_{1}^{0}=-0.035 \log (\kappa)-0.075 \mathrm{~V}$. The effect of chemical kinetics may be hidden if the intermediate is not stable. Fig. 11 shows the change of a single pair of peaks under the influence of diminishing dimensionless rate constant. Dimensionless anodic peak current decreases from 0.675 to 0.393 , but the cathodic peak current changes from -0.230 to -0.265 .

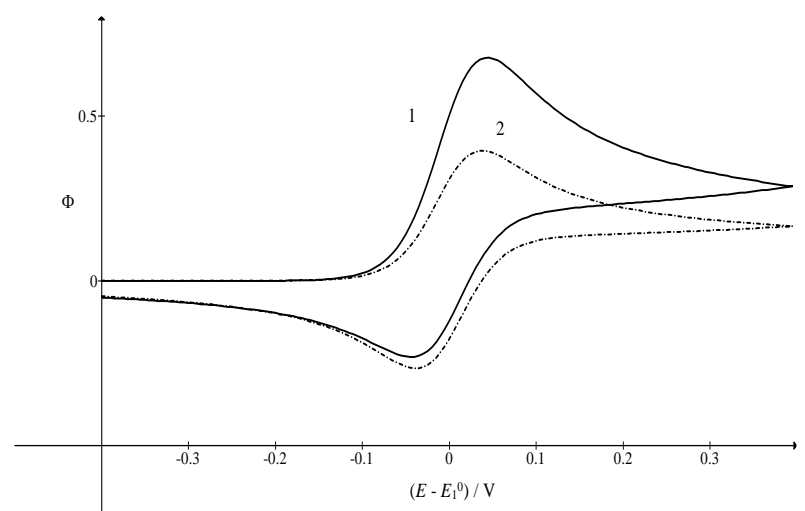

Fig. 11. Cyclic voltammograms of kinetically controlled ECE mechanism. $E_{2}^{o}=E_{1}^{0}$ and $\kappa=1$ (1) and 0.01 (2).

Comparing to Fig. 1, a reversible transfer of single electron that appears for $\kappa=0.01$ turns into irreversible two-electrons electrode reaction for $\kappa=$ 1. In the experiments one can observe that the relationship between peak current and the square root of scan rate is not linear. This is shown in Fig. 12.

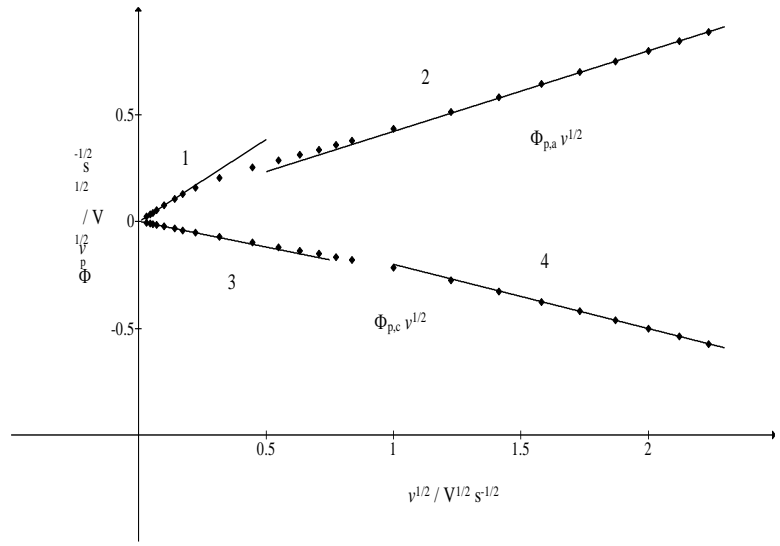

Fig. 12. Dependence of normalized peak currents on the square root of scan rate. ECE mechanism controlled by the kinetics of totally irreversible chemical reaction. $E_{2}^{0}=E_{1}^{0}$ and $k=3 \mathrm{~s}^{-1}$.

The slopes of straight lines 1 and 2 are 0.77 and 0.38 , respectively. The first slope corresponds to two electrons oxidation appearing for higher values of the dimensionless kinetic parameter, while the second one belongs to the single electron oxidation. 
Š. Komorsky-Lovrić, M. Lovrić: Theory of staircase cyclic voltammetry of two electrode reactions ...

However, the slopes of straight lines 3 and 4 are 0.24 and -0.30 because the cathodic current originates from the reduction of either the species $\mathrm{F}^{+}$, at higher $\kappa$ values, or $\mathrm{B}^{+}$at lower $\kappa$ values.

The variation of scan rate may influence the kinetics of both chemical and electrode reactions [5]. An example is shown in Fig. 13. The kinetics of electrode reactions depends on the dimensionless parameters $\lambda_{1}$ and $\lambda_{2}$ (see eqs. 46 and 47 ), but the ratio of electrochemical and chemical kinetic parameters depends on the scan rate: $\lambda_{1} / \kappa=$ $k_{s 1} \sqrt{F v / D R T} / k$.

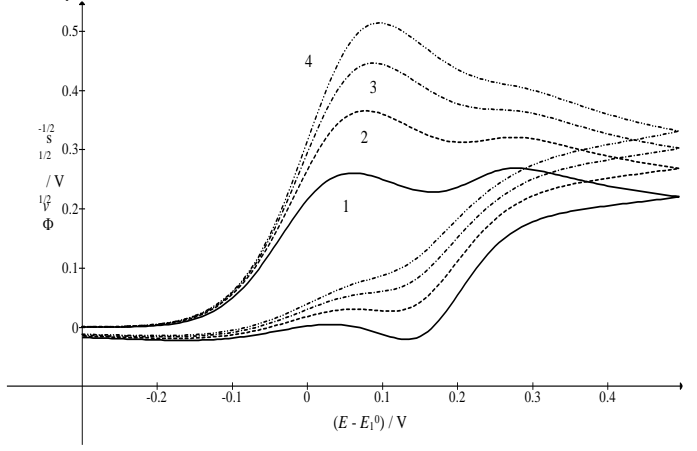

A

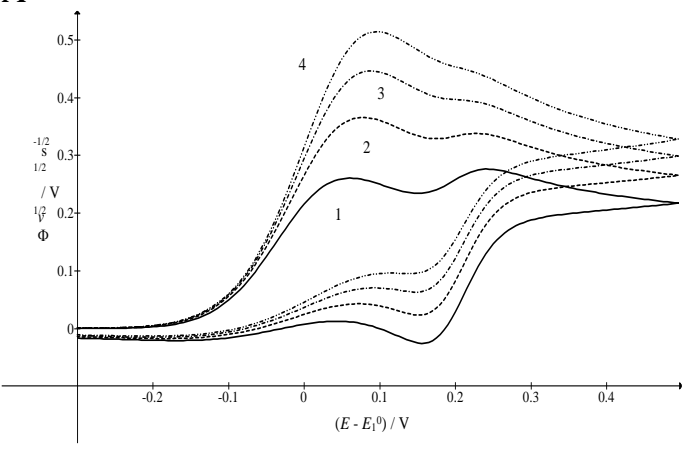

B

Fig. 13. Normalized cyclic voltammograms of ECE mechanism influenced by the kinetics of both chemical and electrode reactions (eqs. 39 and 40). $E_{2}^{0}-E_{1}^{0}=0.2$ $\mathrm{V}, \Delta E=5 \mathrm{mV}, D=9 \times 10^{-6} \mathrm{~cm}^{2} / \mathrm{s}, k=3 \mathrm{~s}^{-1}, \alpha_{1}=0.5, \alpha_{2}=$ $0.5, k_{s 1}=0.01 \mathrm{~cm} / \mathrm{s}, k_{s 2} /(\mathrm{cm} / \mathrm{s})=0.01(\mathrm{~A})$ and $1(\mathrm{~B})$ and $v /(\mathrm{V} / \mathrm{s})=1(1), 2(2), 3(3)$ and $4(4)$.

For this reason Fig. 13 shows the influence of scan rate on the normalized voltammograms. The chemical kinetic parameter decreases with the scan rate as follows: $\kappa=0.0771$ (1), 0.0386 (2), 0.0257 (3) and 0.0193 (4). One can notice that the second anodic peak disappears if the scan rate is higher than $2 \mathrm{~V} / \mathrm{s}$ regardless of the ratio between rate constants of the first and the second electrode reaction. This is in agreement with the critical chemical kinetic parameter $\kappa_{\text {crit }}=0.03$ that is predicted for reversible electrode reactions. Comparing with Fig. 10 , it can be noticed that the anodic peak potentials increase with the scan rate, but two peaks do not overlap if the difference between standard potentials is high enough. However, if these conditions are not satisfied, the critical scan rate cannot be determined. This is shown in Fig. 14. The second anodic peak is diminished with the scan rate because the parameter $\kappa$ decreases from 1.54 , for $v=0.050 \mathrm{~V} / \mathrm{s}$, to 0.0386 for $v=2 \mathrm{~V} / \mathrm{s}$. As the first anodic peak potential increases with the scan rate and the second one does not, two anodic peaks overlap and the second peak disappears at the scan rate that is lower than the theoretical one.

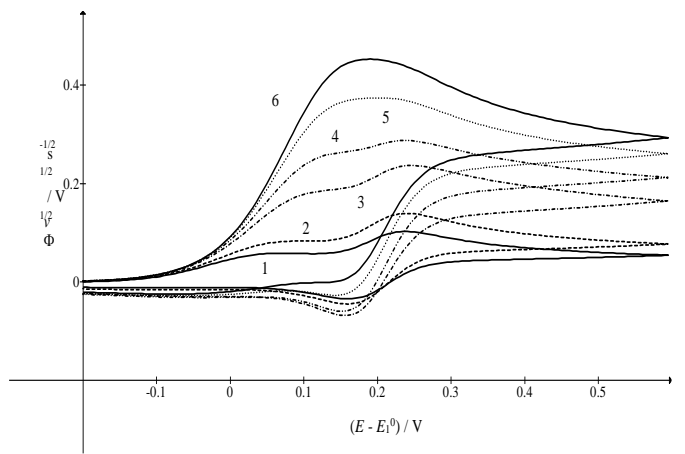

Fig. 14. CV of ECE mechanism (eqs. 39 and 40); $k_{s 1}$ $=0.002 \mathrm{~cm} / \mathrm{s}, k_{s 2}=0.1 \mathrm{~cm} / \mathrm{s}$ and $v /(\mathrm{V} / \mathrm{s})=0.05(1), 0.1$ (2), 0.5 (3), 1 (4), 2 (5) and 3 (6). All other data are as in Fig. 13.

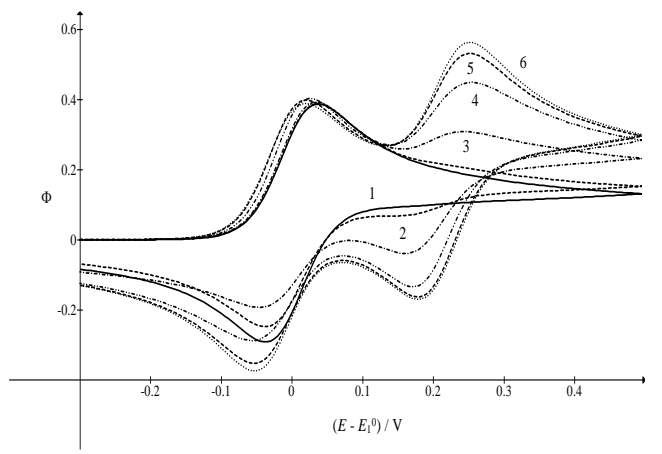

A

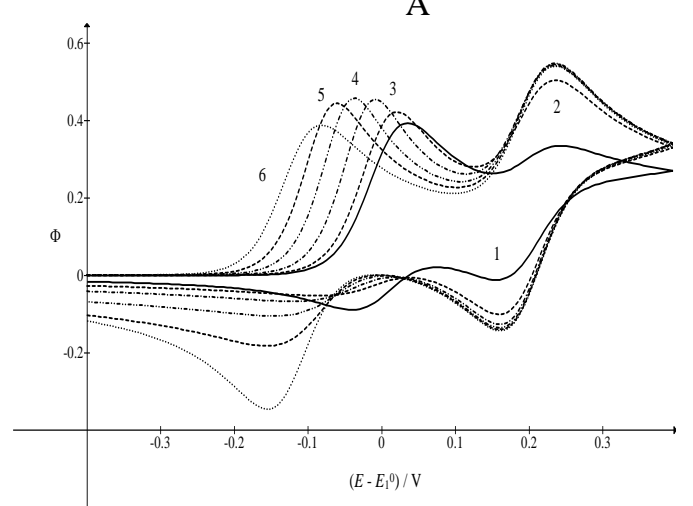

B

Fig. 15. Dimensionless voltammograms of ECE mechanism controlled by the kinetics of reversible chemical reaction (eqs. 53 and 54). $E_{2}^{0}-E_{1}^{0}=0.2 \mathrm{~V}, K c_{X}^{*}$ $=1(\mathrm{~A})$ and $100(\mathrm{~B})$ and $\kappa_{b}=0.001$ (1), 0.01 (2), 0.1 (3), 1 (4), 10 (5) and $\rightarrow \infty$ (6).

The equilibrium constant and the kinetics of the reversible chemical reaction are considered in the last theoretical model. The reaction (2) depends on 
$\check{S}$. Komorsky-Lovrić, M. Lovrić: Theory of staircase cyclic voltammetry of two electrode reactions ... dimensionless rate constants $\kappa_{b}=k_{b} R T / F v$ and $\kappa_{f}=\kappa_{b} K c_{X}^{*}$. The latter corresponds to the dimensionless rate constant $\kappa$ of totally irreversible chemical reaction. The responses depend on $\kappa_{b}$ and $K c_{X}^{*}$, as can be seen in Fig. 15. These parameters can be changed by the variation of scan rate and the concentration of the reactant $\mathrm{X}^{-}$. If $K c_{X}^{*}=1$ the forward and backward kinetic parameters are equal $\left(\kappa_{f}=\kappa_{b}\right)$ and the response tends to the equilibrium conditions with increasing $\kappa_{b}$. The curve 6 is calculated by the first model. The second anodic peak appears if $\kappa_{f, \text { crit }}=0.03$, which means that $k_{f} c_{X}^{*}=1.17 v_{\text {crit }}$. The same was predicted by the second model. Fig. 15B shows that for $K c_{X}^{*}=100$ and $\kappa_{b}=10$ the first electrode reaction appears irreversible and out of equilibrium. Under these conditions the second electrode reaction is in the equilibrium, but the backward chemical reaction limits the first cathodic peak current. This is because the equilibrium is shifted towards the species $\mathrm{E}$ and the value of $\kappa_{b}$ that is required for the approaching to the equilibrium is higher than in Fig. 15A.

The influence of the reagent $\mathrm{X}^{-}$is shown in Fig. 16 for various scan rates. The relationships between the first anodic peak potential and the logarithm of the product $K c_{X}^{*}$ are curves that tend to the asymptotes with the slope $-0.030 \mathrm{~V}$ (see curves $1-$ $4)$. The straight line 5 is calculated by the first model and its slope is $-2.3 R T / F$. The difference in slopes is caused by the fact that the first electrode reaction appears irreversible for the chosen kinetic parameters. This is confirmed by Fig. 17 in which the voltammograms influenced by the variation of the concentration of the reagent $\mathrm{X}^{-}$at the constant scan rate are shown. It can be seen that the first cathodic peak current vanishes with the increasing product $K c_{X}^{*}$. Fig. 18 shows that for the given value of the product $K c_{X}^{*}$ the scan rate has to be decreased as to increase the parameter $\kappa_{b}$ in order to approach the equilibrium conditions. The curve 5 is calculated by the first model.

Generally, the equilibrium constant cannot be measured by the variation of the reactant $\mathrm{X}^{-}$ concentration, but it can be estimated by the simulation using the value of the forward rate constant of chemical reaction that is determined from the critical scan rate at which the second anodic peak appears.

\section{CONCLUSIONS}

Four mathematical models of ECE mechanism are described. The first two models are simplified in order to determine the parameters that can be measured by the cyclic voltammetry.

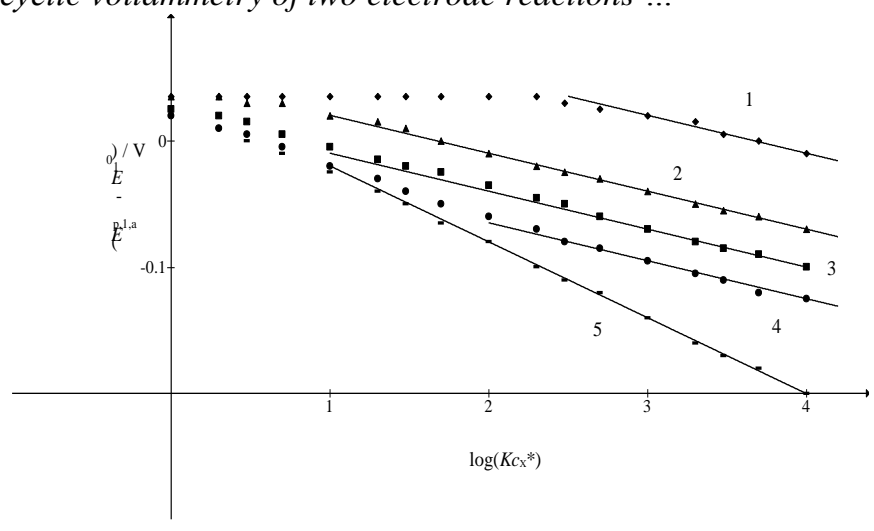

Fig. 16. Dependence of the first anodic peak potential on the logarithm of the dimensionless equilibrium constant of the kinetically controlled reversible chemical reaction. $E_{2}^{0}-E_{1}^{0}=0.2 \mathrm{~V}$ and $\kappa_{b}=0.001$ (1), 0.1 (2) 1 (3), 10 (4) and $\rightarrow \infty$ (5).

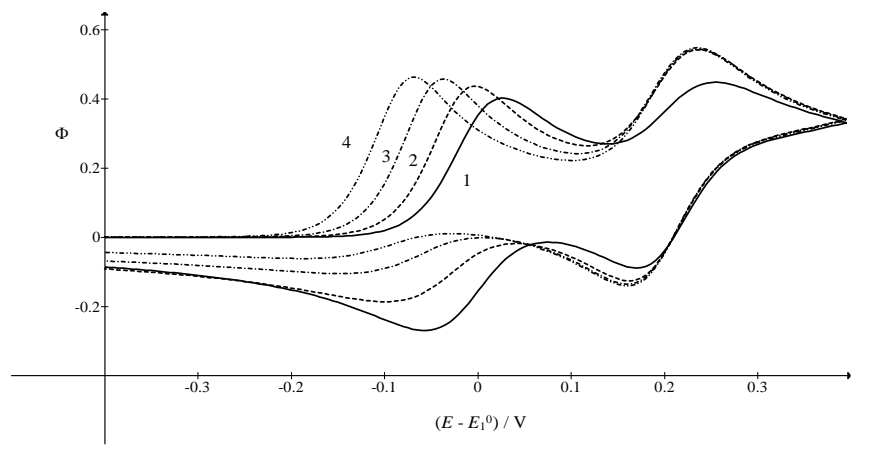

Fig. 17. $\mathrm{CV}$ of ECE mechanism. $E_{2}^{0}-E_{1}^{0}=0.2 \mathrm{~V}, \kappa_{b}$ $=1$ and $K c_{X}^{*}=1$ (1), 10 (2), 100 (3) and 1000 (4).

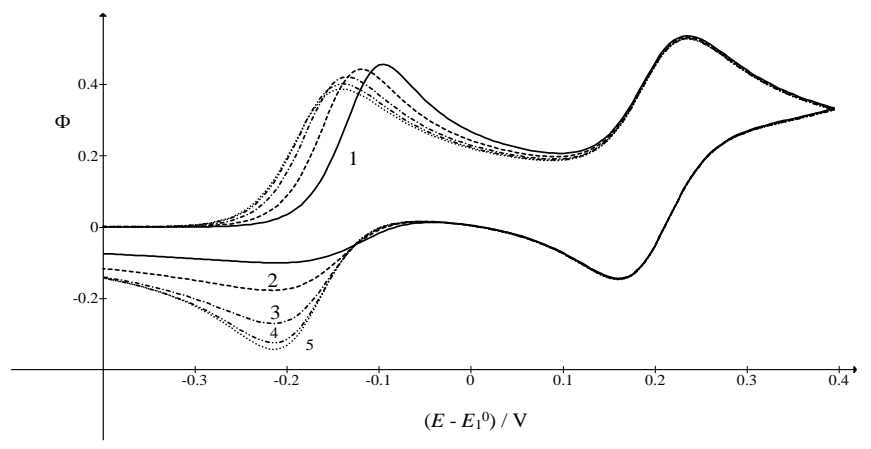

Fig. 18. $C V$ of ECE mechanism. $E_{2}^{0}-E_{1}^{0}=0.2 \mathrm{~V}, K c_{X}^{*}=$ 1000 and $\kappa_{b}=10$ (1), 100 (2), 1000 (3), 10000 (4) and $\rightarrow \infty$ (5).

The other two models are general and they define the conditions under which the first two models are realistic. The simulation demonstrates that the equilibrium constant of chemical reaction can be measured by the variation of the concentration of the reagent $\mathrm{X}^{-}$if the chemical reaction is permanently in equilibrium. Furthermore, the critical kinetic parameter is calculated that enables the measurement of the rate constant of totally irreversible chemical reaction. However, it is shown that the kinetics of electrode reactions may hinder the proper estimation of the chemical rate constant. 
S. Komorsky-Lovrić, M. Lovrić: Theory of staircase cyclic voltammetry of two electrode reactions ...

Finally, the last model shows that the chemical reaction appears irreversible if the concentration of the reagent $\mathrm{X}^{-}$is increased and that the critical kinetic parameter applies under this condition. Also, the determination $\mathrm{n}$ of the equilibrium constant by the fitting procedure is discussed.

\section{REFERENCES}

1. L. Nadjo, J.M. Saveant, J. Electroanal. Chem., 33, 419 (1971).

2. S. W. Feldberg, J. Phys. Chem., 75, 2377 (1971).

3. C. Amatore, J. M. Saveant, J. Electroanal. Chem., 86, 227 (1978).

4. M. J. Palys, M. Bos, W. E. van der Linden, Anal. Chim. Acta, 283, 811 (1993).

5. P. T. Sanecki, C. Amatore, P. M. Skital, J. Electroanal. Chem., 546, 109 (2003).

6. P. T. Sanecki, P. M. Skital, Electrochim. Acta, 53, 7711 (2008).

7. R. Gulaboski, V. Mirčeski, I. Bogeski, M. Hoth, J. Solid State Electrochem., 16, 2315 (2012).

8. J. Jacq, J. Electroanal. Chem., 29, 149 (1971).

9. E. Laviron, R. Meunier-Prest, J. Electroanal. Chem., 324, 1 (1992).

10. R. Gulaboski, V. Markovski, Z. Jihe, J. Solid State Electrochem., 20, 3229 (2016).

11. A. Molina, E. Laborda, J. M. Gomez-Gil, R. G. Compton, J. Solid State Electrochem., 20, 3239 (2016).

12. R.S. Nicholson, I. Shain, Anal. Chem., 37, 190 (1965).

13. G.S. Alberts, I. Shain, Anal. Chem., 35,1859 (1963).

14. B. D. Rossemaar, F. Hartl, D. J. Stufkens, C. Amatore, E. Maisonhaute, J. N. Verpeaux, Organometallics, 16, 4675 (1997).

15. J. W. Hershberger, R. J. Klinger, J. K. Kochi, J. Am. Chem. Soc., 105, 61 (1983).

16. C. C. Neto, S. Kim, Q. Meng, D. A. Sweigart, Y. K. Chung, J. Am. Chem. Soc., 115, 2077 (1993).

17. P. Sanecki, K. Kaczmarski, J. Electroanal. Chem., 471, 14 (1999).

18. P. Sanecki, P. Skital, K. Kaczmarski, Electroanalysis, 18, 981 (2006).

19. S. O'Toole, S. Pentlavalli, A. P. Doherty, J. Phys. Chem. B, 111, 9281 (2007).

20. T. Yamamura, K. Shirasaki, H. Sato, Y. Nakamura, H. Tomiyasu, I. Satoh, Y. Shiokawa, J. Phys. Chem. C, 111, 18812 (2007).

21. S. W. Feldberg, L. Jeftić, J. Phys. Chem., 76, 2439 (1972).

22. R.D. Moulton, D.J. Chandler, A.M. Arif, R.A. Jones, A.J. Bard, J. Am. Chem. Soc., 110, 5714 (1988).

23. Š. Komorsky-Lovrić, M. Lovrić, Collect. Czech Chem. Commun., 72, 1398 (2007).

24. G. J. Wilson, C. Y. Lin, R. D. Webster, J. Phys. Chem. B, 110, 11540 (2006).

25. R. Meng, S. G. Weber, J. Electroanal. Chem., 600, 325 (2007).
26. R. N. Adams, M. D. Hawley, S. W. Feldberg, J. Phys. Chem., 71, 851 (1967).

27. D. Nematollahi, S. M. Golabi, J. Electroanal. Chem., 481, 208 (2000).

28. Y. Li, M. Liu, C. Xiang, Q. Xie, S. Yao, Thin Solid Films, 497, 270 (2006).

29. G. Manning, V. D. Parker, R. N. Adams, J. Am. Chem. Soc., 91, 4584 (1969).

30. M. Fleischmann, F. Lasserre, J. Robinson, J. Electroanal. Chem., 177, 115 (1984).

31. S. O. Engblom, J. C. Myland, K. B. Oldham, Anal. Chem., 66, 3182 (1994).

32. M. D. Hawley, S. W. Feldberg, J. Phys. Chem., 70, 3459 (1966).

33. C. Amatore, J. M. Saveant, J. Electroanal. Chem., 86, 227 (1978).

34. J. Galvez, A. Molina, R. Saura, F. Martinez, J. Electroanal. Chem., 127, 17 (1981).

35. B. Kastening, Anal. Chem., 41, 1142 (1969).

36. H. R. Sobel, D. E. Smith, J. Electroanal. Chem., 26, 271 (1970).

37. M. Mastragostino, L. Nadjo, J. M. Saveant, Electrochim. Acta, 13, 721 (1968).

38. C. Amatore, J. M. Saveant, J. Electroanal. Chem., 85, 27 (1977).

39. R.S. Nicholson, I. Shain, Anal. Chem., 37,178 (1965).

40. M. A. Mann, J. C. Helfrick Jr., L. A. Bottomley, J. Electrochem. Soc., 163, H3101 (2016).

41. A. B. Miles, R. G. Compton, J. Electroanal. Chem., 499, 1 (2001).

42. J. J. O'Dea, K. Wikiel, J. Osteryoung, J. Phys. Chem., 94, 3628 (1990).

43. A.B. Miles, R.G. Compton, J. Phys. Chem. B, 104, 5331 (2000).

44. S̆. Komorsky-Lovrić, M. Lovrić, Anal. Bioanal. Electrochem., 5, 291 (2013).

45. S. Karp, J. Phys. Chem., 72, 1082 (1968).

46. K. B. Prater, A. J. Bard, J. Electrochem. Soc., 117, 1517 (1970).

47. C. Demaille, P. R. Unwin, A. J. Bard, J. Phys. Chem., 100, 14137 (1996).

48. R. Gulaboski, J. Solid State Electrochem., 13, 1015 (2009).

49. R. Gulaboski, P. Kokoškarova, S. Mitrev, Electrochim. Acta, 69, 86 (2012).

50. M. L. Olmstead, R. S. Nicholson, J. Electroanal. Chem., 16, 145 (1968).

51.L.K. Bieniasz, Modelling Electroanalytical Experiments by the Integral Equation Method, Springer, Berlin, 2015.

52. M. Lopez-Tenes, A. Molina, C. Serna, M.M. Moreno, J. Gonzalez, J. Electroanal. Chem., 603, 249 (2007).

53. A. Molina, J. Gonzalez, E. Laborda, R. G. Compton, Int. J. Electrochem. Sci., 7, 5765 (2012).

54. Š. Komorsky-Lovrić, M. Lovrić, Croat. Chem. Acta, 90, 27 (2017).

55. D. DeFord, N. Hume, J. Am. Chem. Soc., 73, 5321 (1951). 